\title{
Potential Myogenous Temporomandibular Disorders Following Iatrogenic Occlusal Disturbance: A Pilot Study
}

\author{
Abdulwahab H Alamir ${ }^{1}$, Yara A Hakami ${ }^{2}$, Fatimah S Alabsi ${ }^{3}$, Esam Halboub ${ }^{4}$, Amal M Bajonaid ${ }^{5}$
}

\begin{abstract}
Aim:There is an inadvertent alteration of the occlusal surface after dental treatment. However, research concerning the effect of these iatrogenic disturbances on the temporomandibular joint (TMJ) is scarce. Hence, the present study aimed to investigate the relationship between sudden iatrogenic occlusal disturbance and its effect on the myogenous temporomandibular disorder (TMD).

Materials and methods: A cross-sectional observational study was conducted among 30 female subjects aged 18 years to 38 years who received treatment (direct and indirect restorations altering the occlusal surfaces of teeth, and oral prophylaxis) in the students' clinics at the College of Dentistry Jazan University. The preoperative assessment included a patient interview, a baseline occlusal record, and bite force analysis. All the assessments were repeated 2 weeks after the treatment. Descriptive statistics were calculated.

Results: As there was no change observed in bite force as well as occlusal disturbances in the control group, no comparative tests could be applied. The treatment-induced occlusal disturbances were observed in $80 \%$ of the participants. The mean change in occlusal force on the treated teeth was found to be $5.6 \pm 1.1$ Newtons. None of the patients reported any symptoms related to pain or restriction in function in the TMJ. Conclusion: No relation was observed between the abrupt changes in the occlusal force due to dental treatment and the development of the myogenous TMD.

Clinical significance: latrogenic occlusal interferences may cause/exacerbateTMDs; hence, extreme caution should be exercised by the clinicians to avoid causing harm to the patients.
\end{abstract}

Keywords: Bite force, latrogenic, Myogenous, Occlusal interference, Temporomandibular disorders.

The Journal of Contemporary Dental Practice (2019): 10.5005/jp-journals-10024-2689

\section{INTRODUCTION}

Temporomandibular disorder comprises a group of chronic pain conditions that can involve the TMJs, masticatory muscles, and associated structures and tissues. ${ }^{1}$ Temporomandibular disorder is most commonly observed in individuals between the ages of 20 years and 40 years. ${ }^{2,3}$ Approximately $33 \%$ of the population has at least one TMDs symptom, and 3-6\% to $7 \%$ of the population has TMD with sufficient severity to cause them to seek treatment. ${ }^{3,4}$ The subtypes of TMDs routinely seen in patients are myofascial pain and arthralgia, followed by disc displacements with reduction. ${ }^{5}$

The TMDs are multifactorial in etiology, as biomechanical, neuromuscular, biopsychosocial, and neurobiological factors may contribute to the disorder. ${ }^{6}$ To highlight their role in the pathogenesis of TMDs, these factors are classified $\mathrm{as}^{4}$

- Predisposing factors (structural, metabolic, and psychological conditions),

- Initiating factors (trauma from occlusion, traumatic injuries), and

- Aggravating factors (parafunctional, psychosocial, or hormonal).

Although the occlusal interferences (OI) had been considered as a significant contributor in characterizing TMD, lately this has been questioned based on epidemiological and experimental studies, and there is a current trend toward making a weak correlation between OI and TMD.?

Recently, the iatrogenic factors resulting from dental practice have also been claimed to be contributing factors in TMD. ${ }^{7}$ Dental treatments affect the shape of the dental units, thus interfering morphologically and functionally with the harmony of the

\begin{abstract}
1,4 Department of Maxillofacial Surgery and Diagnostic Sciences, College of Dentistry, Jazan University, Jazan, Kingdom of Saudi Arabia ${ }^{2}$ College of Dentistry, Jazan University, Jazan, Kingdom of Saudi Arabia ${ }^{3}$ Department of Restorative Dental Sciences, College of Dentistry, Jazan University, Jazan, Kingdom of Saudi Arabia

${ }^{5}$ Department of Oral Medicine, Infection and Immunity, Division of Oral Medicine and Dentistry, Brigham and Women's Hospital, Boston, Massachusetts, USA; Department of Oral Medicine, Infection and Immunity, Harvard School of Dental Medicine, Boston, Massachusetts, USA; Department of Maxillofacial Surgery and Diagnostic Sciences, College of Dentistry, Jazan University, Jazan, Kingdom of Saudi Arabia

Corresponding Author: Abdulwahab $\mathrm{H}$ Alamir, Department of Maxillofacial Surgery and Diagnostic Sciences, College of Dentistry, Jazan University, Jazan, Kingdom of Saudi Arabia, Phone: +966 507633755, e-mail: dr.abdulwahab@hotmail.com
\end{abstract}

How to cite this article: Alamir AH, Hakami YA, Alabsi FS, et al. Potential Myogenous Temporomandibular Disorders Following latrogenic Occlusal Disturbance: A Pilot Study. J Contemp Dent Pract 2019;20(10):1138-1140.

Source of support: Nil

Conflict of interest: None

masticatory system and its components, especially the occlusal condition. ${ }^{8}$ Therefore, the emergence of TMD signs and symptoms after restorative treatment is not rare. It seems that the acute morphological alteration of the occlusal conditions as a result of restorative or prosthodontic restoration may interfere with the established functional equilibrium, disturbing the coordinated pattern and timing of jaw muscle contraction. ${ }^{7}$ 
Most restorative procedures affect the shape of the occlusal surface; however, the iatrogenic effect of dental restoration in TMD etiology has rarely been studied. Hence, this pilot study was aimed to investigate the relationship between sudden iatrogenic occlusal disturbance and its effect on the myogenous TMD.

\section{Materials and Methods}

\section{Study Design}

A cross-sectional observational study was conducted to investigate the relationship between sudden iatrogenic occlusal disturbance and its effect on the myogenous TMD among patients who received treatment at the students' clinic at the College of Dentistry Jazan University.

\section{Study Population}

The study population consisted of 30 female subjects aged 18 years to 38 years who received treatment (direct and indirect restorations altering the occlusal surfaces of teeth, and oral prophylaxis) in the students' clinics at the College of Dentistry Jazan University. The patients were selected on the following inclusion and exclusion criteria.

\section{Inclusion Criteria}

- Saudi nationality, and

- No known health disparities at the time of enrolment in the study.

\section{Exclusion Criteria}

- The patients suffering from any dental problem, for example, loss of posterior support, unilateral posterior cross-bite, patient with extreme overjet and overbite, cracked tooth syndrome, occlusal trauma, and moderate-to-severe periodontal disease, and patients with the history of TMJ disorders were excluded.

\section{Ethical Clearance and Informed Consent}

The ethical approval for the study was obtained from the Institutional Review Board of Jazan University. Written informed consent was obtained from the participants after explaining the study protocol in the local language.

\section{Study Protocol}

The research consisted of three groups: two study groups (operative and fixed prosthesis) and one comparison group (oral prophylaxis).

The preoperative assessment included a patient interview to answer questions about pain or restriction in function in the TMJ and a baseline record using the T-Scan III computerized occlusal analysis system (T-Scan, Inc., South Boston, MA, USA). Also, bite force analysis was done for all the participants. The subjects then received their treatment according to the scheduled procedure with the student.

Two weeks after receiving the treatment, the subjects were interviewed again for any pain or restriction in function, and the second record of their occlusion was taken using the T-Scan. Additionally, bite force analysis was done 2 weeks after the treatment. No clinical examination was conducted.

\section{Statistical Analysis}

The data were entered in Microsoft Office, Excel worksheets, and analyzed using the software IBM SPSS v. 20.0 (IBM Statistics; SPSS,
Chicago, IL, USA). The normality of the data was assessed using the Shapiro-Wilk test while Levene's test for equality of error variances was used to analyze the homogeneity of error variances. Descriptive statistics were calculated. As there was no change observed in bite force as well as occlusal disturbances in the control group, no comparative tests could be applied.

\section{Results}

A total of 30 female patients with a mean age of $24 \pm 5.3$ years were included in the present pilot study. The study participants were divided into three groups, namely:

Group I: patients receiving direct occlusal restoration (operative clinic) total of 10 patients (33.33\%).

Group II: patients receiving indirect occlusal restoration (fixed prosthesis clinic) 2 patients (6.67\%).

Group III: patients receiving oral prophylaxis (periodontal clinic) 18 patients (60\%).

The distribution of subjects was $40 \%$ in the treatment group, and $60 \%$ in the control group.

The treatment-induced occlusal disturbances were observed in $8(80 \%)$ of the participants in group I and all the patients in group II, whereas no occlusal disturbances were found in the control group.

Furthermore, the mean change in occlusal force on the treated teeth was found to be $5.6 \pm 1.1$ Newtons. This change in occlusal force was not statistically significant $(p>0.05)$. As expected, no change in bite force was observed in the control group. Also, none of the patients reported any symptoms related to pain or restriction in function in the TMJ. Hence, the results recorded no relation between the abrupt changes in the occlusal force due to dental treatment and the development of the myogenous TMD.

\section{Discussion}

The present study was conducted to examine the relation, if any, between OI resulting from dental treatment and mygenous TMDs. It was observed that a very high percentage of dental treatments resulted in the creation of occlusal disturbances, and there was a slight change in the bite force in the restored dentition. However, this change was not statistically significant, and it did not produce any symptoms related to TMJ.

The first point to ponder is the alarmingly high percentage of occlusal disturbances introduced after the treatment. It could be because the procedures were performed in a student clinic rather than by experienced dentists. There is a significant difference in the quality of the restoration produced by a skilled practitioner and an amateur. However, in spite of the large number of $\mathrm{Ol}$ in the restored teeth, none of the patients reported any symptoms related to the TMJ. Furthermore, the mean change in the bite force produced after the restorative treatment was also negligible. These findings further weaken the argument favoring the relationship between Ol and TMDs.

The capacity of the masticatory muscles to exert sufficient bite force between the teeth is an indicator of normal function. ${ }^{9}$ In patients with TMD, there is a diminished capacity of the masticatory muscle activity. As a result, the maximal bite force values are generally lower in patients with TMD than in the population in general. ${ }^{9,10}$ Hence, bite force was used as an indicator of TMD in the present.

The etiology of TMDs is multifactorial, and various authors have emphasized the importance of occlusion. However, whether 
occlusion plays a crucial role in the pathogenesis of TMD remains controversial. This argument is based on various epidemiological and experimental studies, and there is a current trend toward making a weak correlation between OI and TMD. It had been reported that 85 to 90 percent of the population exhibit some form of OI. ${ }^{7,11}$ Other studies conducted in different population groups with different ages and gender have shown that various types of occlusal disturbances were significantly but often weakly correlated with the signs and symptoms of TMD.,12,13 Furthermore, experimental studies have also reached controversial results. Some studies have demonstrated no electromyographic response to the artificial $\mathrm{OI}^{14,15}$ whereas others showed that occlusal disturbances were significantly associated with consistent clinical changes in the electromyographic response as well as clinical symptoms. ${ }^{16-18}$

The findings of the present study are contrasting to those of a 10-year controlled longitudinal clinical study that researched the probable etiological significance of restorative treatment leading to TMD among 40 participants. It showed that signs and symptoms of TMD were more evident in subjects with restored dentition than in subjects with intact dentition. ${ }^{19}$ This is generally attributed to inappropriately contoured fillings leading to occlusal instability. Furthermore, over-contoured anterior crowns without adequate lingual concavity will direct the jaw along a retruded closing path, forcing the condyle onto a more sensitive vascular innervation area. This results in the condyles to be more distally placed leading to hyperactivity and fatigue in posterior temporalis and the suprahyoid muscles and deep masseter muscles, thereby resulting in inter-articular disc displacement. ${ }^{7}$

Nevertheless, occlusal interference can cause TMJ dysfunction. Uncoordinated mandibular movements seem to be one of the neuromuscular responses of the masticatory system to Ol. Restorative procedures aim to alleviate TMJ dysfunction by eliminating as many $\mathrm{Ol}$ as possible. However, despite the best efforts and technical skill of the clinicians, Ol can be inadvertently incorporated in the restored occlusal morphology. ${ }^{20}$

\section{Limitations and Recommendations}

It can be argued that the sample size in the present study was too small to produce significant result. Hence, it is recommended to conduct future research taking a larger and more homogeneous study population to study the influence of iatrogenic occlusal disturbances. Also, the study revealed a glaring gap in the students' clinical skills. Therefore, the academicians should work on improving the clinical training of students so that they do not cause iatrogenic problems for the patients.

\section{Conclusion}

Within the limitations, it can be concluded from the present study that:

- Occlusal disturbance after operative and fixed treatment among patients treated in students' clinic was significantly high.

- A change in the occlusal force of 5.6 Newton did not produce any symptoms indicative of TMJ disorders.

\section{Clinical Significance}

latrogenic OI may cause/exacerbate TMDs; hence, extreme caution should be exercised by the clinicians to avoid causing harm to the patients.

\section{References}

1. McNeill C, Mohl ND, Rugh JD, et al. Temporomandibular disorders: diagnosis, management, education, and research. J Am Dent Assoc 1990;120:253, 255, 257, 10.14219/jada.archive.1990.0049.

2. Gesch $D$, Bernhardt $O$, Alte $D$, et al. Prevalence of signs and symptoms of temporomandibular disorders in an urban and rural German population: results of a population-based Study of Health in Pomerania. Quintessence Int 2004;35:143-150.

3. Wright EF. Manual of temporomandibular disorders. Ames (IA): Wiley; 2005. pp. 60-111.

4. Xie Q, Li X, Xu X. The difficult relationship between occlusal interferences and temporomandibular disorder-insights from animal and human experimental studies. J Oral Rehabil 2013;40(4): 279-295. DOI: 10.1111/joor.12034.

5. LeResche L. Epidemiology of temporomandibular disorders: implications for the investigation of etiologic factors. Crit Rev Oral Bio Med 1997 Jul;8(3):291-305. DOI: 10.1177/10454411970080030401.

6. Suvinen TI, Reade PC, Hanes KR, et al. Temporomandibular disorder subtypes according to self-reported physical and psychosocial variables in female patients: a re-evaluation. J Oral Rehabil 2005;32:166-173. DOI: 10.1111/j.1365-2842.2004.01432.x.

7. Hagag G, Yoshida K, Miura H. Occlusion, prosthodontic treatment, and temporomandibular disorders: a review. J Med Dent Sci 2000;47(1):61-66.

8. Bengt O. Prosthodontics, Principles and Management Strategies. London: Mosby-Wolfe; 1996. p. 97.

9. Kogawa EM, Calderon PS, Lauris JR, et al. Evaluation of maximal bite force in temporomandibular disorders patients. J Oral Rehabil 2006; 33(8):559-565. DOI: 10.1111/j.1365-2842.2006.01619.x.

10. Garner LD, Kotwal NS. Correlation study of incisive biting forces with age, sex, and anterior occlusion. J Dent Res 1973;52:698-702. DOI: 10.1177/00220345730520041001.

11. Agerberg G, Sandstorm R. Frequency of occlusal interference: a clinical study in teenagers and young adults. J Prosthet Dent 1988;59:212-217. DOI: 10.1016/0022-3913(88)90017-0.

12. Solberg WK, Woo MW, Houston JB. Prevalence of mandibular dysfunction in young adults. J Am Dent Assoc 1979;98:25-34. DOI: 10.14219/jada.archive.1979.0008.

13. Ingervall B, Mohlin B, Thilander B. Prevalence of symptoms of functional disturbances of the masticatory system in Swedish men. J Oral Rehabil 1980;7:185-197. DOI: 10.1111/j.1365-2842.1980. tb00435.x.

14. Schaerer P, Stallard RE, Zander HA. Occlusal interferences and mastication: an electromyographies study. J Prosthet Dent 1967;17:438-449. DOI: 10.1016/0022-3913(67)90141-2.

15. DeBoever J. Experimental occlusal balancing contact interferences and muscle activity. An electromyographies study with permanently applied electrodes. Parodontologie 1969;23:59-69.

16. Sheikholeslam A, Riise C. Influence of experimental interfering occlusal contacts on the activity of the anterior temporal and masseter muscles during submaximal and maximal bite in the intercuspal position. J Oral Rehabil 1983;10:207-214. DOI: 10.1111/ j.1365-2842.1983.tb00114.x.

17. Rugh JD, Barghi N, Drago CJ. Experimental occlusal discrepancies and nocturnal bruxism. J Prosthet Dent 1984;51:548-553. DOI: 10.1016/0022-3913(84)90312-3.

18. Magnusson T, Enbom L. Signs and symptoms of mandibular dysfunction after introduction of experimental balancingside interference. Acta Odontol Scand 1984;42:129-135. DOI: 10.3109/00016358408993863.

19. Kamp T, Hannerz H, Storm P. Ten-year follow up study of signs and symptoms of craniomandibular disorders in adults with intact and restored dentitions. J Oral Rehabil 1996;23:416-423. DOI: 10.1111/ j.1365-2842.1996.tb00873.x.

20. Michelotti A, lodice $G$. The role of orthodontics in temporomandibular disorders. J Oral Rehabil 2010;37(6):411-429. DOI: 10.1111/j.13652842.2010.02087.x. 